\title{
Modelos teóricos del capital humano y señalización: un estudio para México
}

\section{Theoretical models of human capital and signaling: a study for Mexico}

\author{
Antonio Kido Cruz ${ }^{\mathrm{a}, *}$ y M. Teresa Kido Cruz ${ }^{\mathrm{b}}$ \\ ${ }^{\text {a } U n i v e r s i d a d ~ M i c h o a c a n a ~ d e ~ S a n ~ N i c o l a ́ s ~ d e ~ H i d a l g o, ~ F a c u l t a d ~ d e ~ C o n t a d u r i ́ a ~ y ~ C i e n c i a s ~ A d m i n i s t r a t i v a s, ~ M e ́ x i c o ~}$ \\ b Universidad del Papaloapan, Campus Oaxaca, México
}

Recibido el 19 de febrero de 2014; aceptado el 2 de junio de 2014

Disponible en Internet el 14 de agosto de 2015

\section{Resumen}

El marco de referencia más usual que asigna un papel preponderante a la formación educativa para el fortalecimiento en el bienestar y el crecimiento económico lo representa el modelo del capital humano. Sin embargo, el enfoque teórico de la señalización implica que un proceso de mayor apoyo a la educación puede tener sus limitantes cuando se trata de generar impactos en el crecimiento macroeconómico. El objetivo de este estudio fue el de investigar, si la evidencia empírica disponible en México sobre niveles de escolaridad y rendimientos a la educación responde a un modelo de capital humano o a un modelo de señalización. Se utilizó un modelo macroeconómico y microeconómico de corrección de errores con información de series de tiempo. Los resultados señalan que para el caso de México la información disponible favorece un modelo de capital humano.

Derechos Reservados (C) 2015 Universidad Nacional Autónoma de México, Facultad de Contaduría y Administración. Este es un artículo de acceso abierto distribuido bajo los términos de la Licencia Creative Commons CC BY-NC-ND 4.0.

\section{Palabras clave: Capital humano; Señalización; Crecimiento económico}

\section{Abstract}

The most common reference framework which assigns a prominent role to education for strengthening welfare and economic growth is represented by the human capital model. However, the theoretical approach

\footnotetext{
* Autor para correspondencia.

Correo electrónico: akido42@ hotmail.com (A. Kido Cruz).

La revisión por pares es responsabilidad de la Universidad Nacional Autónoma de México.
} 
of signaling implies that a process of greater support for education may have its limitations when it comes to generating impacts on macroeconomic growth. The aim of this study was to investigate if the empirical evidence available in Mexico on levels of schooling and returns to education responds to a human capital model or a model of signaling. A macroeconomic and a microeconomic model error correction were used. The results show that for the case of Mexico available information favors a model of human capital.

All Rights Reserved (c) 2015 Universidad Nacional Autónoma de México, Facultad de Contaduría y Administración. This is an open access item distributed under the Creative Commons CC License BY-NC-ND 4.0.

Keywords: Human capital; Signalization; Economic growth

\section{Introducción}

Un tema ampliamente debatido en el ámbito de la economía de la educación ha sido la eficacia de la educación formal para aumentar la productividad individual y el crecimiento económico de un país. La herramienta más frecuentemente utilizada, la ecuación de salarios, ha sido empleada para contrastar la teoría del capital humano (Schultz, 1960; Becker, 1962 y Mincer, 1958). De acuerdo a esta teoría la educación supone que una mayor inversión en capital humano aumenta la productividad de los trabajadores y, por tanto, su salario así como el crecimiento económico.

Sin embargo, ha surgido una explicación alternativa, que explica la asociación positiva entre educación y salarios. Esta señala que la educación tiene como principal objetivo el proporcionar información sobre algunas características de los potenciales trabajadores a los dueños de las empresas (Arrow, 1973; Spence, 1973; Stiglitz, 1956) y se conoce como la teoría de la señalización.

La dirección de la relación causal entre la capacidad salarial y la educación tiene importantes implicaciones de política educativa. De la teoría del capital humano se desprende que las políticas incentivadoras de la educación y la formación de los ciudadanos de niveles de bajos ingresos son las adecuadas para conseguir una distribución más igualitaria entre los trabajadores de diferentes estratos y mayor crecimiento económico. Sin embargo, si la educación es considerada solo como una señal, la acumulación de capital humano no se traduciría forzosamente en un incremento de la productividad de la fuerza de trabajo. Podría incluso pensarse, en este caso, que las inversiones educativas no generan un beneficio para la economía.

De acuerdo a Raymond y Barceinas (1999) las dos teorías son empíricamente equivalentes pero sus implicaciones prácticas son opuestas a nivel de la sociedad. Es por eso que se vuelve necesaria la verificación de cualquiera de las teorías a través de los enunciados observacionales disponibles. En este sentido, la práctica común a nivel internacional, para contrastar estas teorías se ha basado en estudios de sección cruzada. Podemos señalar el modelo de escolaridad y genero de Mora y Muro (2012), la contrastación de salarios entre autoempleados y trabajadores a sueldo en diferentes sectores y segmentos (Fields, 2011); la inclusión de una variable aproximada del nivel de habilidades (Acemoglu y Autor, 2012); la estrategia de medir la evolución de la desigualdad entre empleados y la polarización del empleo en economía avanzadas (Brynjolfsson y McAfee, 2013).

En este estudio nos interesa proponer un contraste entre la hipótesis del capital humano versus la hipótesis de señalización utilizando evidencia observacional longitudinal que contrasta con todos los estudios anteriores, a través de un modelo de corrección de errores. 
El artículo está dividido en cinco partes: la primera de ellas corresponde a la introducción y la segunda hace referencia a la información relevante en el tema para el caso de México. En la sección tres se comenta el marco teórico. La sección cuatro desarrolla los modelos empíricos de estimación y se analizan las fuentes de información utilizada. La sección cinco contiene los resultados de los modelos y la conclusión principal del trabajo.

\section{Información relevante en México}

Aunque existe una cantidad abundante de estudios que han medido la relación entre ingresos y educación en México (Carnoy, 1967; Bracho y Zamudio, 1994; Psacharopoulos, Velez, Panagides y Yang, 1996; Singh y Santiago, 1997, Smith y Metzer, 1998; Barceinas, 1999; Berhman et al., 1999; Binder, 1999; Rojas, Angulo y Velázquez, 2000; Taylor y Yunez-Naude, 2000; Carnoy, 2006; Barceinas, 2002; Huesca, 2004; Mehta y Villarreal, 2005; Varela y Retamoza, 2011 y Varela y Retamoza, 2012) no todos han hecho énfasis en contrastar si esta relación se explica más desde el contexto del modelo de capital humano o del modelo de señalización.

De los estudios señalados en el párrafo anterior, Barceinas (2002) concluyen que para el caso mexicano los resultados obtenidos en su estudio no son favorables a la hipótesis de señalización. En primer lugar, esta sugeriría que los rendimientos de la educación de los autoempleados y del sector privado deberían ser significativamente menores a los correspondientes de los asalariados y del sector público, respectivamente. En general, los autoempleados tienen rendimientos muy similares a los asalariados y los rendimientos de los trabajadores del sector privado son mayores que los del sector público. Concluyen que aunque el modelo de señalización explica una parte del comportamiento del mercado laboral mexicano, es el modelo de capital humano el que mayormente determina la relación entre salarios y sus diferencias.

Por otra parte, Mehta y Villarreal (2005) concluyen que existe evidencia empírica a favor del modelo de señalización solo en el caso de los trabajadores con educación primaria y en el ámbito urbano, lo que no sucede para el caso de los trabajadores del sector rural.

De esta manera, el objetivo principal de este trabajo es el de contrastar la hipótesis del modelo de capital humano con la del modelo de señalización para el caso mexicano con información de series de tiempo. La estrategia es estimar un modelo con información macroeconómica con la inclusión de la variable de escolaridad (de aquí en adelante se le llamará modelo macrominceriano) y estimar un modelo con datos macroeconómicos (incluyendo la variable de la rentabilidad educativa y a la cual llamaremos microminceriano) y obtener su respectiva participación porcentual.

A priori, en el modelo que sustenta la teoría del capital humano, el coeficiente estimado para la variable «escolaridad» y la variable «rentabilidad educativa» deberán coincidir. Esto indicaría que una mayor educación en lo particular generará gente más productiva, lo que aumentará el ingreso de las personas y en consecuencia, una mayor educación en promedio generará una nación más productiva, por lo que habría que esperar un aumento en el ingreso nacional. En contraposición, en un modelo de señalización puro, se espera que el coeficiente de rentabilidad educativa en la especificación microminceriana tienda a ser positivo y el coeficiente de la variable escolaridad en el modelo macrominceriano tienda a cero (por lo menos en el margen).

\section{Bases teóricas del modelo de Solow ampliado}

En este trabajo, se utilizará el modelo de crecimiento económico aumentado de Solow sin descuidar la relación conjunta entre el nivel tecnológico y la aportación del capital humano. Para 
incorporar la variable del capital humano (Mankiw, Romer y Weil, 1992) parten de considerar una función de producción tipo Cobb-Douglas:

$$
Y_{t}=K_{t}^{\alpha} H_{t}^{\beta}\left(A_{t} L_{t}\right)^{1-\alpha-\beta}
$$

En donde Y representa el producto nacional; K es el nivel de capital agregado en la economía; $\mathrm{H}$ representa el factor del capital humano; A es el factor tecnología y L corresponde al agregado laboral. En el estado estacionario, tanto la acumulación del capital físico como del capital humano están determinados por la siguiente expresión:

$$
\begin{aligned}
& \Delta k_{t}=s_{k} y_{t}-(n+g+\delta) k_{t} \\
& \Delta h_{t}=s_{h} y_{t}-(n+g+\delta) h_{t}
\end{aligned}
$$

Y en estado estacionario:

$$
\begin{aligned}
k^{*} & =\left(\frac{s_{k}^{1-\beta} s_{h}^{\beta}}{(n+g+\delta}\right)^{\left(\frac{1}{1-\alpha-\beta}\right)} \\
h^{*} & =\left(\frac{s_{k}^{\alpha} s_{h}^{1-\alpha}}{(n+g+\delta}\right)^{\left(\frac{1}{1-\alpha-\beta}\right)}
\end{aligned}
$$

Sustituyendo estos valores en la función de producción y expresándola en términos per cápita, nos queda:

$$
\begin{gathered}
\ln \left(Y_{t}^{*} / L_{t}\right)=\ln A_{0}+g t+\frac{\alpha}{1-\alpha-\beta} \ln s_{k}+\frac{\beta}{1-\alpha-\beta} \ln s_{h} \\
-\frac{\alpha-\beta}{1-\alpha-\beta} \ln (n+g+\delta)
\end{gathered}
$$

Esta ecuación muestra como el ingreso per cápita depende del crecimiento demográfico y la acumulación del capital físico y humano.

\section{El modelo empírico}

Para evitar el problema de multicolinealidad de los datos se estandariza el producto y la acumulación de capital en unidades de trabajo y se asume que la elasticidad de escala de los factores de la producción es igual a la unidad. Se especifica una función de producción con componentes logarítmicos en su forma estructural como:

$$
\log y_{t}=\log A_{t}+\alpha \log k_{t}+\beta \log h_{t}+\mu_{t}
$$

Donde las letras en minúsculas representan $\mathrm{y}=\mathrm{Y} / \mathrm{L}$ representa el producto por trabajador; $\mathrm{k}=\mathrm{K} / \mathrm{L}$ se refiere al capital físico en términos intensivos y $\mathrm{h}=\mathrm{H} / \mathrm{L}$ que representa la acumulación promedio del capital humano. Las series macroeconómicas fueron sometidas a pruebas de raíz unitaria presentando los resultados de las mismas en la siguiente tabla. Los resultados del análisis se basaron en la prueba de Augmented Dickey-Fuller (ADF), la prueba de Phillis-Perron (PP) y la prueba de Kwiatowski-Phillips-Smicht-Shin (KPSS). La prueba de ADF y PP se basa en que la hipótesis nula establece que las respectivas series de tiempo son estacionarias en diferencia 
mientras que la prueba de raíz unitaria de KPSS se basa en la hipótesis nula que establece que las series de tiempo son estacionarias en tendencia.

El número de rezagos utilizados fue de 1 y el criterio de información analizado corresponde al de Aikake. Todas las variables presentan integración de orden (1).

Los resultados de la prueba de raíz unitaria para las series macroeconómicas sugieren la elección de un modelo de corrección de errores que combina información de largo plazo con un mecanismo de ajuste de corto plazo. El modelo de corrección de errores puede estimarse de diversas maneras, entre ellas la sugerida por Banerjee, Dolado, Galbraith y Hendry, 1993. Este procedimiento muestra que el modelo de corrección de errores de una etapa generalizado representa un modelo autorregresivo con distribución de rezagos y por lo tanto, puede utilizarse para estimar la relación de series que contienen procesos no estacionarios.

De esta manera y siguiendo el enfoque de que las variables menos significativas se van eliminando (Hendry, 1995) es posible especificar el modelo de corrección de errores que incluye una variable de capital humano (escolaridad) en la función de producción aumentada de Solow como:

$$
\begin{aligned}
\Delta \log y_{t}= & \gamma_{1} \Delta \log k_{t}+\gamma_{2} \Delta \log k_{t-1} \\
& \quad-\gamma_{3}\left(\log y_{t-1}-\alpha \log k_{t-1}-\beta \log h_{t-1}-\log A_{t-1}\right)+\mu_{t}
\end{aligned}
$$

Donde $\Delta$ representa la primera diferencia o tasa de crecimiento de las series y Vt es el vector de errores con las propiedades usuales. Cualquier técnica de regresión estándar es válida para estimar la ecuación (8) ya que todos los términos son estacionarios cuando se toma su primera diferencia. El coeficiente $\gamma_{3}$, que representa el término de corrección de error indica el periodo de ajuste hacia el equilibrio de largo plazo y los parámetros $\gamma_{1}$ y $\gamma_{2}$ capturan los efectos de corto plazo sobre sus respectivos regresores, que al estar las series referidas en logaritmos también representan las elasticidades corto plazo.

Se asume que la acumulación del capital humano solo tiene efectos sobre el producto en el largo plazo, por lo que la ecuación anterior se convierte de la forma:

$$
\begin{aligned}
\Delta \log y_{t}=c & +\gamma_{1} \Delta \log k_{t}+\gamma_{2} \Delta \log k_{t-1}+\gamma_{3} \log y_{t-1}+\gamma_{4} \log k_{t-1}+\gamma_{5} \log h_{t-1} \\
& +\sum j \delta_{j} \text { comercio }_{j, t}+\mu_{t}
\end{aligned}
$$

La cual puede ser estimada mediante la técnica de mínimos cuadrados ordinarios. Los estimadores sobre el parámetro de $\gamma_{3}$ pueden ser utilizados para calcular las elasticidades $\alpha$ y $\beta$ de la ecuación (7). Adicionalmente, se interpreta que en un escenario de estimación de una sola ecuación, un coeficiente significativo, en términos estadísticos, proporciona una prueba de cointegración (Banarjee, Dolado y Mestre, 1998).

De acuerdo a Loening (2004) el coeficiente A de la ecuación (1) que se refiere a que la tecnología puede variar a lo largo del tiempo, se puede representar como una función de $\mathrm{Z}$ en donde $\log A_{t}=f\left(Z_{t}\right)$ el cual, en su expresión más simple puede ser aproximado por la constante c y una variable ficticia (comercio). En nuestro caso se considera una variable estructural de los términos de intercambio comercial dentro del tratado de libre comercio de América del Norte.

Con la finalidad de contrastar la participación de la acumulación en el capital humano en el crecimiento económico en México del modelo anterior, es posible proponer un modelo macroeconómico que incorpore un indicador microeconómico del capital humano (rentabilidad educativa), 
con lo cual se estaría estimando un modelo de tipo minceriano. Se parte de la especificación de Bils y Klenow (2000):

$$
Y_{t}=K_{t}^{\alpha}\left[A_{t} H_{t}\right]^{(\alpha-1)}
$$

Donde Y representa el nivel de producto, K es la acumulación física del capital, A representa un índice tecnológico y $\mathrm{H}$ es el capital humano. De acuerdo a estos autores, la acumulación agregada del capital humano representa la suma del capital humano por cohorte de trabajo y se asume que toda cohorte va a la escuela desde la edad 0 hasta la edad s (por tanto, el nivel de estudios está representado por s) y empieza a trabajar desde s hasta la edad T. De esta manera, es posible especificar que:

$$
H_{t}=\int_{s}^{T} h(a, t) L(a, t) d a
$$

En donde $L(a, t)$ representa el número de trabajadores de la cohorte a en el período t y $h(a, t)$ el nivel del capital humano. Generalizando para el caso en que s y $\mathrm{T}$ difieran para las diferentes cohortes de trabajadores, es posible señalar que la acumulación del capital humano en forma individual se expresa como:

$$
h_{t}=h(a+n, t) \varphi e^{f(s)+g(a-s)} \quad \text { para todo } \mathrm{a}>\mathrm{s}
$$

El parámetro $\varphi$ es el mayor interés en esta ecuación ya que captura la influencia del capital humano. Se destaca el caso cuando $\varphi$ es igual a cero, ya que en estas condiciones, $\mathrm{f}(\mathrm{s})=\theta \mathrm{s}$ y $\mathrm{g}$ (a $\mathrm{s})=\gamma_{1}(\mathrm{a}-\mathrm{s})+\gamma_{2}(\mathrm{a}-\mathrm{s})^{2}$. De esta manera la ecuación (12) se reduce a la ecuación que especificó Mincer en 1974. Esta especificación implica que el logaritmo del salario per cápita se relaciona, linealmente, con los años de escolaridad, los años de experiencia y los años de experiencia al cuadrado de un individuo en particular.

Siguiendo la misma estrategia de especificación econométrica que en la ecuación (1), nos queda:

$$
\log y_{t}=\log A_{t}+\alpha \log k_{t}+(1-\alpha) \phi h_{t}
$$

La función de producción se transforma en un modelo de corrección de errores en una etapa, quedando:

$$
\begin{aligned}
\Delta \log y_{t}= & \gamma_{1} \Delta \log k_{t}+\gamma_{2} \Delta \log k_{t-1} \\
& -\gamma_{3}\left(\log y_{t-1}-\alpha \log k_{t-1}-(1-\alpha) \phi h_{t-1}-\log A_{t-1}\right)+\mu_{t}
\end{aligned}
$$

Finalmente, reparametrizando el modelo anterior para incluir el efecto de las variables ficticias, nos queda:

$$
\begin{aligned}
\Delta \log y_{t}=c & +\gamma_{1} \Delta \log k_{t}+\gamma_{2} \Delta \log k_{t-1}+\gamma_{3} \log y_{t-1}+\gamma_{4} \log k_{t-1}+\gamma_{5} h_{t-1} \\
& +\sum j \delta_{j} \text { comercio }_{j, t}+\mu_{t}
\end{aligned}
$$




\section{Compilación de información}

Las principales series estadísticas requeridas para la estimación se refieren a la acumulación del capital humano, la acumulación del capital físico, el producto interno bruto, el volumen del comercio exterior con relación al producto interno bruto y la fuerza laboral. Todas estas series con excepción de la variable de capital humano se obtuvieron de los indicadores del Banco Mundial para México (Banco Mundial, 2013). Las series fueron convertidas en términos per cápita y están representadas en dólares reales con año base 2005 y abarcan el período de 1980 al año 2012.

El capital humano es una variable difícil de medir en su dimensión exacta. La variable más utilizada se refiere a los años promedio de escolaridad. Estas series las maneja el Instituto Nacional de Estadística Geografía e Informática (2011) ya que presenta una serie de años promedio de escolaridad en forma anual desde el año de 1980 hasta el año 2009 y esta es la proxy de capital humano utilizada en este estudio.

Para generar la variable proxy de capital humano en el modelo microminceriano fue necesario conseguir información sobre la rentabilidad educativa en México en forma anual. La serie más completa la presenta Varela y Retamoza (2012) en donde estima tasas de retorno educativo en México para los años de 2000 al 2009. Con información de la Encuesta Nacional de Ingreso Gasto (ENIGH-INEGI) fue posible estimar las tasas de rendimiento educativo para México en los años 1984, 1989, 1992, 1994, 1996, 1998 y 2000. Finalmente, los datos de la inversión educativa para los años 1991, 1993, 1994, 1995, 1997 y 1999 para México se obtuvieron de informe de la Organización del trabajo (OIT, 2013).

Las variables utilizadas para la estimación minceriana se obtienen de los archivos de las variables por hogar e ingresos y percepciones del hogar de la Encuesta Nacional de Ingreso Gasto (varios años) del Instituto de Información, Geografía e Informática. Estos archivos contienen información sobre la edad, la escolaridad y el nivel de sueldos del jefe del hogar (tabla 1).

Tabla 1

Prueba de raíz unitaria

\begin{tabular}{|c|c|c|c|c|c|c|}
\hline Variable & $\mathrm{ADF}(\mathrm{C})$ & $\mathrm{ADF}(\mathrm{C}+\mathrm{T})$ & $\mathrm{PP}(\mathrm{C})$ & $\mathrm{PP}(\mathrm{C}+\mathrm{T})$ & KPSS (C) & $\operatorname{KPSS}(\mathrm{C}+\mathrm{T})$ \\
\hline In ingreso per cápita & -0.33 & -1.52 & -1.92 & -2.76 & 1.07 & 0.25 \\
\hline $\begin{array}{l}(\mathrm{y}) \\
\Delta \ln \mathrm{y}\end{array}$ & -5.33 & -4.49 & -17.80 & -8.12 & 0.32 & 0.17 \\
\hline In escolaridad (h) & -2.7 & -3.11 & -1.06 & -2.41 & 1.51 & 0.24 \\
\hline$\Delta \ln \mathrm{h}$ & -3.68 & -5.08 & -20.29 & -12.78 & 0.25 & 0.09 \\
\hline ln capital (k) & -2.37 & -2.52 & -2.51 & -2.16 & 1.41 & 0.23 \\
\hline$\Delta \ln \mathrm{k}$ & -5.57 & -4.81 & -8.63 & -7.80 & 0.34 & 0.05 \\
\hline In comercio & -1.89 & -2.56 & -2.06 & -2.52 & 1.34 & 0.29 \\
\hline$\Delta \ln$ comercio & -4.29 & -7.79 & -8.76 & -7.80 & 0.25 & 0.11 \\
\hline $\begin{array}{l}\text { In rentabilidad } \\
\text { educativa }(\Phi \text { h) }\end{array}$ & -1.97 & -2.09 & -1.96 & -2.85 & 1.57 & 0.31 \\
\hline$\Delta \ln \Phi \mathrm{h}$ & -4.74 & -5.09 & -9.91 & -4.57 & 0.20 & 0.10 \\
\hline
\end{tabular}

Valores críticos para la prueba de raíz unitaria de ADF y la prueba PP considerando solo la constante: a (1\%) -3.47, b (5\%), -2.88, y c (10\%) -2.57. Los valores de la prueba de raíz unitaria incluyendo solo la constante son: a (1\%) 0.739 , b (5\%) 0.463 y, c (10\%) 0.347. Los valores críticos de la prueba de raíz unitaria considerando constante y tendencia de ADF y PP son: a (1\%) -4.02\%, b (5\%)-3.44 y, c (10\%) -3.14. Los valores críticos de la prueba de raíz unitaria considerando constante y tendencia para KPSS son: a (1\%) 0.74 , b (5\%) $0.46 \mathrm{y}, \mathrm{c}(10 \%) 0.34$.

Fuente: Elaboración propia. 
Tabla 2

Resumen de estadísticas descriptiva de los datos

\begin{tabular}{lcclc}
\hline & $\mathrm{y}$ & $\mathrm{h}$ & $\mathrm{k}$ & comercio \\
\hline Media & 5.0931875 & 6.859375 & $1.2708 \mathrm{E}+11$ & 46.2215752 \\
Error típico & 0.47843233 & 0.23442876 & $1.77891 \mathrm{E}+9$ & 2.57557295 \\
Mediana & 4.3475 & 7.05 & $1.1859 \mathrm{E}+11$ & 53.575119 \\
Desviación estándar & 2.70642196 & 1.32612931 & $4.4062 \mathrm{E}+10$ & 14.5696408 \\
Varianza de la muestra & 7.32471983 & 1.75861895 & $1.9415 \mathrm{E}+21$ & 212.274432 \\
Rango & 8.595 & 4.3 & $1.4083 \mathrm{E}+11$ & 41.0509934 \\
Mínimo & 1.681 & 4.5 & $7.1296 \mathrm{E}+10$ & 23.3444926 \\
Máximo & 10.276 & 8.8 & $2.1212 \mathrm{E}+11$ & 64.395486 \\
Suma & 162.982 & 219.5 & $4.0664 \mathrm{E}+12$ & 1479.09041 \\
Cuenta & 22 & 22 & 22 & 22 \\
\hline
\end{tabular}

Fuente: elaboración propia.

Tabla 3

Regresión lineal macroeconómica del producto per cápita y capital humano en México 1980-2010 (ecuación 9)

\begin{tabular}{lll}
\hline Variable & Coeficiente & Error estándar \\
\hline Constante & 0.0932 & 0.0104 \\
$\Delta$ ln k & 0.0016 & 0.00078 \\
ln k_1 & 0.0076 & 0.0066 \\
ln y_1 & -0.00979 & -0.107 \\
ln h_1 & 0.00330 & 0.00182 \\
Ln variable ficticia & $3.20 \mathrm{E}-15$ & $2.00 \mathrm{E}-14$ \\
Variable de tendencia & & \\
Elasticidad h de largo plazo & 0.337 & \\
$\mathrm{R}^{2}$ & 0.66 & \\
$\mathrm{D}-\mathrm{W}$ & 2.081 & \\
\hline
\end{tabular}

Fuente: elaboración propia.

\section{Resultados empíricos de la estimación}

La tabla 2 muestra el resumen de la descripción estadística básica de los datos utilizados.

La tabla 3 muestra las estimaciones de la ecuación (9) por la técnica de mínimos cuadrados ordinarios. La primera columna identifica las variables empleadas en la estimación. La segunda y tercera columna señalan los coeficientes y el error estándar de la misma ecuación.

La estimación puntual para el coeficiente del capital humano fue de 0.0039 con un error estándar de 0.0018 y una estimación de $R^{2}$ e 0.66 . El valor del parámetro asociado al capital humano representa la elasticidad en el corto plazo, para conocer la elasticidad en el largo plazo se divide el valor del parámetro asociado al capital humano entre el valor del parámetro del producto per cápita. Esto es, el cociente del valor del cambio porcentual del parámetro del capital per cápita dividido por el coeficiente respectivo del capital físico y del capital humano contenidos en $\gamma 3$ representa la elasticidad de largo plazo del capital físico y del capital humano. El resultado para el capital humano nos muestra un coeficiente de $0.33(0.0033 / 0.00979)$ que nos indica que un año adicional de educación, en el promedio nacional, contribuye en la generación de un $33 \%$ en el producto nacional.

Para conocer la robustez estadística de los parámetros estimados, se generaron pruebas estadísticas sobre los residuales de la ecuación (9) y los resultados se muestran en la tabla 4. 
Tabla 4

Pruebas de autocorrelación, heterocedasticidad y significación conjunta sobre los residuales de la ecuación (9)

\begin{tabular}{lcc}
\hline $\begin{array}{l}\text { Pruebas de autocorrelación, heterocedasticidad y } \\
\text { significación conjunta }\end{array}$ & \multicolumn{2}{c}{ Residuales del modelo macromiceriano. Ecuación (9) } \\
\cline { 2 - 3 } & Estadístico de prueba & Valor crítico \\
\hline Prueba Jarque-Bera & 3.77 & 3.29 \\
Prueba de Q-jung & 6.18 & 22.74 \\
Prueba Breusch-Godfrey & 0.31 & 0.84 \\
Prueba de White con producto cruzado & 29.13 & 39.14 \\
Prueba F & 59.02 & 0.037 \\
Reset de Ramsey & 1.31 & 0.22 \\
\hline
\end{tabular}

Tabla 5

Regresión lineal macroeconómica del producto per cápita y capital humano en México 1980-2010 (ecuación 9) con inclusión de una variable de tendencia

\begin{tabular}{lll}
\hline Variable & Coeficiente & Error estándar \\
\hline Constante & 0.1527 & 0.765 \\
$\Delta$ ln k & 0.0021 & 0.00053 \\
ln k_1 & 0.007 & 0.0016 \\
ln y_1 & -0.0096 & -0.104 \\
ln h_1 & 0.0035 & 0.0015 \\
Ln variable ficticia & $3.10 \mathrm{E}-15$ & $2.10 \mathrm{E}-14$ \\
Variable de tendencia & 0.0967 & 0.498 \\
Elasticidad h de largo plazo & 0.29 & \\
$\mathrm{R}^{2}$ & 0.54 & \\
$\mathrm{D}-\mathrm{W}$ & 2.120 & \\
\hline
\end{tabular}

Las pruebas de bondad estadística nos señalan que la especificación de la ecuación (9) no pasa la prueba de la distribución normal de los residuales ya que el valor del estadístico de prueba es menor al valor crítico $(3.77>3.22)$. Asimismo, presenta inconsistencias en la prueba de Reset de Ramsey ya que el valor de la probabilidad de cometer el error de tipo I, es del $22 \%$.

Los resultados anteriores generaron la necesidad de estimar un modelo que incluyera una variable de tendencia para saber si algunas perturbaciones en el tiempo estaban originando inconsistencias estadísticas. Los resultados se muestran en la tabla 5

Este modelo nos indica que el valor del coeficiente del capital humano se calculó en 0.035 con un error estándar de 0.0015 con un R2 de 0.54. La elasticidad en el largo plazo del coeficiente de capital humano $(0.0035 / 0.0097=0.36)$ nos indicaría que un año promedio adicional en la población repercutirá en un incremento del $36 \%$ en el producto nacional por persona. Este modelo supera las pruebas de normalidad, correlación y heterocedasticidad. Sin embargo, la prueba de Reset de Ramsey indica que existe una probabilidad del $20 \%$ de cometer el error I en la especificación del modelo (tabla 6). No obstante los resultados de esta prueba estadística, es importante mencionar que la especificación de las variables del modelo responde a la guía teórica del enfoque del crecimiento económico comentado en la sección del desarrollo teórico.

La tabla 7 presenta los resultados para la ecuación microminceriana. La primera columna presenta las variables de la ecuación (15) y la segunda y tercera columna representan los coeficientes y el error estándar de la misma ecuación. La segunda columna señala un valor de $\beta$ de 0.0017 con un valor de error estándar de 0.00082 y un $R^{2}$ e 0.75 . La elasticidad en el largo plazo se estimó 
Tabla 6

Pruebas de autocorrelación, heterocedasticidad y significación conjunta sobre los residuales de las ecuación (9) con variable de tendencia

\begin{tabular}{lcc}
\hline $\begin{array}{l}\text { Pruebas de autocorrelación, heterocedasticidad y } \\
\text { significación conjunta }\end{array}$ & \multicolumn{2}{c}{ Residuales de la ecuación (9) con variable de tendencia } \\
\cline { 2 - 3 } & Estadístico de prueba & Valor crítico \\
\hline Prueba Jarque-Bera & 2.25 & 3.29 \\
Prueba de Q-jung & 9.58 & 23.6 \\
Prueba Breusch-Godfrey & 0.17 & 0.76 \\
Prueba de White con producto cruzado & 22.15 & 37.47 \\
Prueba F & 6.73 & 0.0371 \\
Reset de Ramsey & 1.19 & 0.20
\end{tabular}

Tabla 7

Regresión lineal microeconómica del producto per cápita y capital humano en México 1980-2010 (ecuación 15)

\begin{tabular}{lll}
\hline Variable & Coeficiente & Error estándar \\
\hline Constante & 0.0927 & 0.0104 \\
$\Delta$ ln k & 0.1833 & 0.0463 \\
$\Delta$ ln k_1 & 0.0053 & 0.0045 \\
$\Delta$ ln y_1 & 0.0137 & 0.0041 \\
Ln $\Phi$ h_1 & 0.0017 & 0.0008 \\
Ln vficicia & $4.20 \mathrm{E}-15$ & $1.38 \mathrm{E}-14$ \\
Elasticidad $\Phi$ h de largo plazo & 0.135 & \\
$\mathrm{R}^{2}$ & 0.75 & \\
$\mathrm{D}-\mathrm{W}$ & 1.9198 & \\
\hline
\end{tabular}

Fuente: elaboración propia.

Tabla 8

Pruebas de autocorrelación, heterocedasticidad y significación conjunta sobre los residuales de las ecuación (15)

Pruebas de autocorrelación, heterocedasticidad y $\quad$ Residuales del modelo microminceriano. Ecuación (15) significación conjunta

\begin{tabular}{lcc} 
& Estadístico de prueba & Valor crítico o valor de probabilidad \\
\hline Prueba Jarque-Bera & 1.96 & 3.29 \\
Prueba de Q-jung & 7.58 & 22.9 \\
Prueba Breusch-Godfrey & 0.16 & 0.88 \\
Prueba de White con producto cruzado & 19.27 & 29.41 \\
Prueba F & 5.17 & 0.035 \\
Reset de Ramsey & 7.09 & 0.009 \\
\hline
\end{tabular}

en $0.135(0.0017 / 0.013)$ que nos indica que un año más de educación en la población nacional incrementa el producto en un $13.5 \%$.

Para comprobar la robustez estadística de las estimaciones, se generaron diferentes pruebas estadísticas sobre autocorrelación, heterocedasticidad y de correcta especificación de los modelos que se resumen en el tabla 8.

Los resultados de la pruebas de bondad estadística nos señalan que la especificación de la ecuación (15) supera las pruebas de normalidad, correlación, heterocedasticidad y de especificación por lo que nos sugiere que este modelo es una mejor representación estadística y al mismo tiempo no limita el contraste teórico del modelo presentado. 
Los resultados del modelo a corto plazo en ambas ecuaciones establecen un coeficiente significativo para la variable del capital humano, con lo que se indica un proceso de cointegración entre las variables incluidas. Dividiendo los coeficientes de la elasticidad de largo plazo de las variables aproximadas al capital humano de los dos modelos $(0.135 / 0.33)$ o $(0.135 / 0.36)$ se obtiene la participación porcentual de la variable de interés en ambos modelos. Contrastando los coeficientes estimados para la variable de capital humano en los modelos obtenidos, la participación porcentual se estimó aproximadamente en (40/60) o (38/42), mostrando en ambos casos un resultado que favorece en mayor grado al modelo de capital humano.

\section{Conclusiones}

Para interpretar los resultados que se presentan en este estudio, es importante destacar que el procedimiento empleado para la contrastación de hipótesis es un mecanismo ad hoc (como ya se ha señalado anteriormente, debido a que las hipótesis no son mutuamente excluyentes) y las tasas de rentabilidad educativa que se han utilizado responden a un contexto específico y restrictivo por la escasez de información en la variable del rendimiento educativo anual para la serie de tiempo. Sin embargo, es posible afirmar que los resultados capturan, en particular, el rendimiento económico esperado de un año adicional de escolarización en un contexto macroeconómico y microeconómico que permiten señalar el sentido y la magnitud de las mismas. Bajo este contexto es posible concluir que existe evidencia empírica de que el modelo de capital humano presente la mayor explicación en el comportamiento de la relación entre escolaridad e ingresos personales en México como lo señalan la gran cantidad de estudios generados en México. La aportación principal de este trabajo radica en que se presenta un mecanismo adicional a los ya existentes para contrastar la hipótesis del modelo del capital humano versus la hipótesis del modelo de señalización. Nuestro estudio utiliza series de tiempo, modelo de corrección de errores y comparación entre coeficientes en contraste con el resto de los enfoque que utilizan información y modelos de sección cruzada.

\section{Referencias}

Acemoglu, D. y Autor, D. (2012). What does capital human do? Journal of Economic Literature, (50), 426-463.

Arrow, K. (1973). Higher education as a filter. Journal of Publics Economics, 2(3), 193-216.

Barceinas, F. (1999). Función de ingresos y rendimiento de la educación en México. Estudios Económicos, 14(15), 87-128.

Barceinas, F. (2002). Rendimientos privados y sociales de la educación en México. Economía Mexicana. Nueva Época, XI(2), 333-390.

Banco Mundial (2013). Indicadores para México. Disponible en http://datos.bancomundial.org/indicador Recuperado el 17 de noviembre de 2013

Banerjee, A., Dolado, J., Galbraith, J. y Hendry, D. (1993). Cointegration, error correction, and the econometric analysis of non-stationary data. Oxford University Press.

Banarjee, A., Dolado, J. y Mestre, R. (1998). Error-correction mechanism test for cointegration in a single-equation framework. Journal of Time Series Analysis, 19(3), 267-283.

Becker, G. (1962). Investment in human capital: a theorical analysis. Journal of Political Economy, (70), 9-49.

Bils, M. y Klenow, P. (2000). Does schooling cause growth? American Economic Review, 90(5), 1160-1183.

Binder, M. (1999). Schooling indicators during Mexico's lost decade. Economics of Education Review, (18), $183-199$.

Bracho, T. y Zamudio, A. (1994). Los rendimientos económicos de la escolaridad en México, 1989. Economía Mexicana, (3), 345-377.

Brynjolfsson, E. y McAfee, A. (2013). La carrera contra la máquina. Antoni Bosch.

Carnoy, M. (1967). Earnings and schooling in Mexico. Economic development and cultural change julio:, $408-418$.

Carnoy, M. (2006). Economía de la educación. Editorial UOC.

Encuesta Nacional de Ingresos Gasto, (2015) (ENIGH- varios años). Archivos por hogar e ingreso. Disponible en www.inegi.com. Recuperado el 19 de noviembre de 2013 
Fields, G. (2011). Labor market analysis for developing countries. Labor Economics, (18), S16-S22.

Hendry, D. (1995). Dynamic Econometrics. Oxford University Press.

Instituto Nacional de Estadística, Geografía e Informática (2011). Mexico desde un Vistazo. Disponible en: www.inegi.org.mx/prod_serv/contenidos/espanol/./mex_2011. Recuperado el 17 de noviembre de 2013

Loening, J. (2004). Time series evidence on education and growth: the case of Guatemala, 1951-2002. Revista de Análisis Económico, 9(2), 3-40.

Mankiw, G., Romer, D. y Weil, D. (1992). A contribution to the empirics of economic growth. Quarterly Journal of Economics, 107(2), 407-437

Mehta, A. y Villarreal, H. (2005). Sheepskins effects in Mexico: evidence with attention to worker heterogeneity. Mimeo.

Mincer, J. (1958). Investment in human capital and personal income distribution. Journal of Political economy, 66(4), 281-302.

Mora, J. y Muro J. (2012). Diploma earnings differnces by genre in Colombia. Disponible en: http://www.icesi. edu.co/ jjmora/pdfs/diploma_earning_differences_by_gender\%20and\%20cohort.pdfRecuperado el 10 de noviembre de 2013

Organización internacional del trabajo (2013). Disponible en: http://www.ilo.org/global/lang-es/index.htm Recuperado el 10 de noviembre de 2013

Psacharopoulos, G., Velez, E., Panagides, A. y Yang, H. (1996). Returns to education during economic boom and recession: Mexico. Education Economics, 4(3), 219-230.

Rojas, M., Angulo, H. y Velázquez, I. (2000). Rentabilidad de la inversión en capital humano en México. Economía Mexicana, IX(2), 113-142.

Singh, R. y Santiago, M. (1997). Farm earnings, educational attainment, and role of public policy: some evidence from Mexico. World Development, 25(12), 2143-2154.

Smith, P. A. y Metzer, M. R. (1998). The return to education: street vendors in Mexico. World Development, 26(2), 289-296.

Spence, M. (1973). Job market signaling. Journal of labour economics, (87), 355-374.

Stiglitz, J. (1956). The theory of screening, education, and the distribution of income. The American Economic Review, (65), 283-300.

Taylor, J. y Yunez-Naude, A. (2000). The returns of schooling in a diversified rural economy. American Journal of Agricultural Economics, (82), 287-297.

Varela, R. y Retamoza, A. (2011). Los salaries en México: un análisis con datos de panel. Investigación y Ciencia, (53), 29-38.

Varela, R. y Retamoza, A. (2012). Capital humano y diferencias salariales en México, 2000-2009. Estudios Fronterizos, (3), 175-200. 